\title{
Seroprevalence of SARS-CoV2 in Qatar: a longitudinal epidemiological study
}

Farah Shurrab ${ }^{1}$, Hadeel Al-Jighefee ${ }^{1}$, Salma Younes ${ }^{1}$, Duaa Al-Sadeq ${ }^{1}$, Asmaa Althani ${ }^{1,2}$, Hadi Yassine ${ }^{1}$, Mohamed Syed ${ }^{3}$, Ahmed Al Nuaimi ${ }^{3}$, Hamda Abdulla ${ }^{3}$, Gheyath Nasrallah ${ }^{1,2}$

1 Biomedical research center (BRC), 2 Department of Biomedical sciences, college of Health Science, 3 Primary Health Care Corporation

\section{Abstract}

In Qatar, the first COVID-19 cases were reported on the $29^{\text {th }}$ of February 2020, and the numbers increased dramatically within few months. Primary Health Care Corporation in collaboration with Qatar University's Biomedical Research Centre initiated a three-phase longitudinal epidemiological study to obtain precise estimates of point and period prevalence by age, gender and nationality. The first phase of the study is done and included 2084 individuals. The results showed that the overall prevalence was $11.1 \%$. However, the point prevalence and the period prevalence of SARS-CoV2 was 1.6 and $9.5 \%$ resp. IgG prevalence was higher amongst 18-39-year old $(10 \%)$ and $\geq 60(11.9 \%)$, and higher in males $(11.6 \%)$ compared with females $(6.2 \%)$, and higher incidences were reported among Highest amongst North African (10.9\%) and Southern Asian $(15.1 \%)$ nationalities.

\section{Objective}

- To conduct a three-phase longitudinal epidemiological study to obtain a precise estimate of SARS-CoV-2 seroprevalence by age, gender and nationality that represent the national Qatar's general population

\section{Introduction}

The novel coronavirus rapidly spread across the globe resulting in a formidable outbreak. Soon, the World Health Organization (WHO) announced the outbreak as a public health emergency of international concern in January 2020. In Qatar, the first COVID-19 cases were reported on the $29^{\text {th }}$ of February 2020, and the numbers increased dramatically within few months. In July 2020, Primary Health Care Corporation in collaboration with Qatar University's Biomedical Research Centre initiated a threephase longitudinal epidemiological study to obtain a precise seroprevalence estimate in Qatar.

\section{Methodology}

Study design and population A 3 phases study, 3-months time interval between each phase . A stratified random sampling technique was utilized. 16 strata were defined using age, gender nationality.

\section{(1) \\ Participant recruitment Eligible participants were extracted from PHCC's electronic medical records. Participants were sent SMS to participate in the study}

Data collection and analysis Nasal and oropharyngeal swab and blood samples were collected.

Samples were analyzed using RT-PCT for active infection and $\mathrm{IgG}$ serology for previous infection using Mindray CL-900i automated analyzer

\begin{tabular}{|c|c|c|c|c|}
\hline & \multicolumn{2}{|c|}{$\begin{array}{l}\text { Active COVID-19 Infection (RT- } \\
\text { PCR) }\end{array}$} & \multicolumn{2}{|c|}{$\begin{array}{l}\text { Prevalence of CoVID-19 Infection } \\
\text { (Blood lgG) }\end{array}$} \\
\hline & $\begin{array}{l}\text { Positive Test } \\
\text { Results }\end{array}$ & Total Tested & Positive Test Results & Total Testec \\
\hline & $N(\%)$ & $\mathrm{N}$ & $\mathrm{N}(\%)$ & $\mathrm{N}$ \\
\hline $10-17$ & $5(1.4)$ & 356 & $25(7.1)$ & 352 \\
\hline $18-39$ & $17(1.9)$ & 918 & $91(10)$ & 913 \\
\hline $40-59$ & $10(1.5)$ & 659 & $56(8.5)$ & 661 \\
\hline$\geq 60$ & 0 & 128 & $15(11.9)$ & 126 \\
\hline Female & $14(1.5)$ & 953 & $59(6.2)$ & 949 \\
\hline Male & $18(1.6)$ & 1108 & $128(11.6)$ & 1103 \\
\hline Qatar & $3(0.6)$ & 486 & $19(3.9)$ & 485 \\
\hline Northern Africa & $3(0.7)$ & 412 & $45(10.9)$ & 412 \\
\hline $\begin{array}{l}\text { South-eastern } \\
\text { Asia }\end{array}$ & $2(1.4)$ & 139 & $11(8)$ & 138 \\
\hline Southern Asia & $14(3)$ & 470 & $57(15.1)$ & 376 \\
\hline $\begin{array}{l}\text { Western Asia } \\
\text { (excluding } \\
\text { Qatar) }\end{array}$ & $7(1.8)$ & 381 & $48(5.6)$ & 861 \\
\hline $\begin{array}{l}\text { Northern } \\
\text { America }\end{array}$ & $1(3.1)$ & 32 & $2(6.1)$ & 33 \\
\hline Europe & $1(1.1)$ & 88 & $1(1.1)$ & 87 \\
\hline Others & $1(1.9)$ & 53 & $4(7.5)$ & 53 \\
\hline Ad-Dawhah & $10(1.3)$ & 752 & $79(10.6)$ & 748 \\
\hline Al Rayyan & $14(2.1)$ & 660 & $52(7.9)$ & 655 \\
\hline Al Daayen & $0(0)$ & 117 & $4(3.4)$ & 116 \\
\hline Umm Salal & $2(1.5)$ & 130 & $9(6.9)$ & 130 \\
\hline Al Khor & $3(6.1)$ & 49 & $5(10.2)$ & 49 \\
\hline Al Shamal & $0(0)^{*}$ & 5 & $1(20)^{*}$ & 5 \\
\hline Al-Shahaniya & $0(0)$ * & 17 & $4(23.5) *$ & 17 \\
\hline Al Wakrah & $1(0.5)$ & 221 & $16(7.2)$ & 221 \\
\hline
\end{tabular}

1. Prevalence and active COVID-19 infection by age.

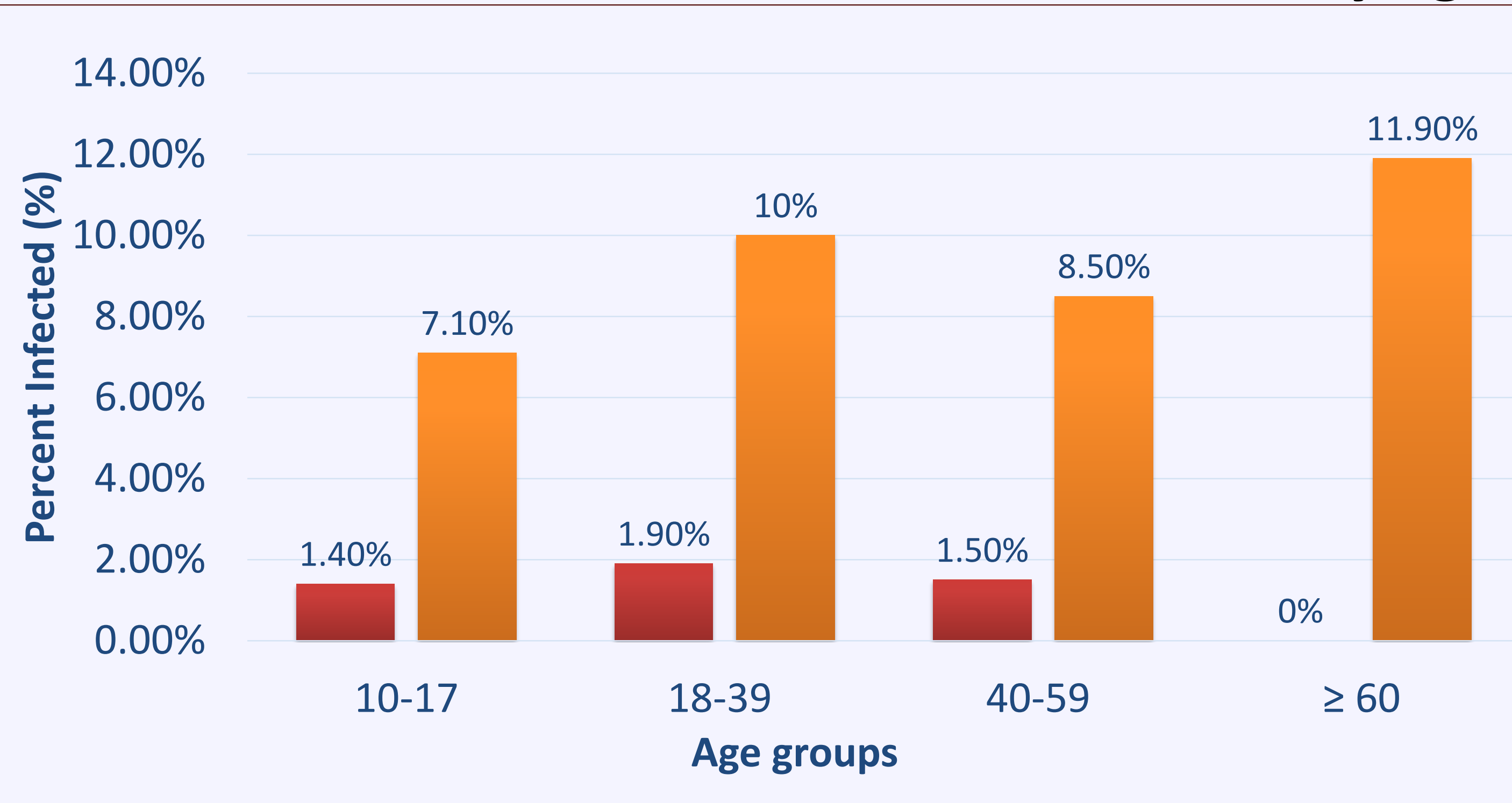

- Active COVID-19 Infection (RT-PCR) 1 Prevalence of COVID-19 Infection (Blood IgG)

Figure 1: this figure shows the percentage of COVID-19 prevalence and active infection among age groups. Active COVID-19 infection was almost similar across all age groups (1.5-1.9\%) except for except $\geq 60(0$ $\%$ ). Prevalence of COVID-19 (IgG positive) was higher amongst 18-39year olds $(10 \%)$ and $\geq 60(11.9 \%)$

2. Prevalence and active COVID-19 infection by gender.

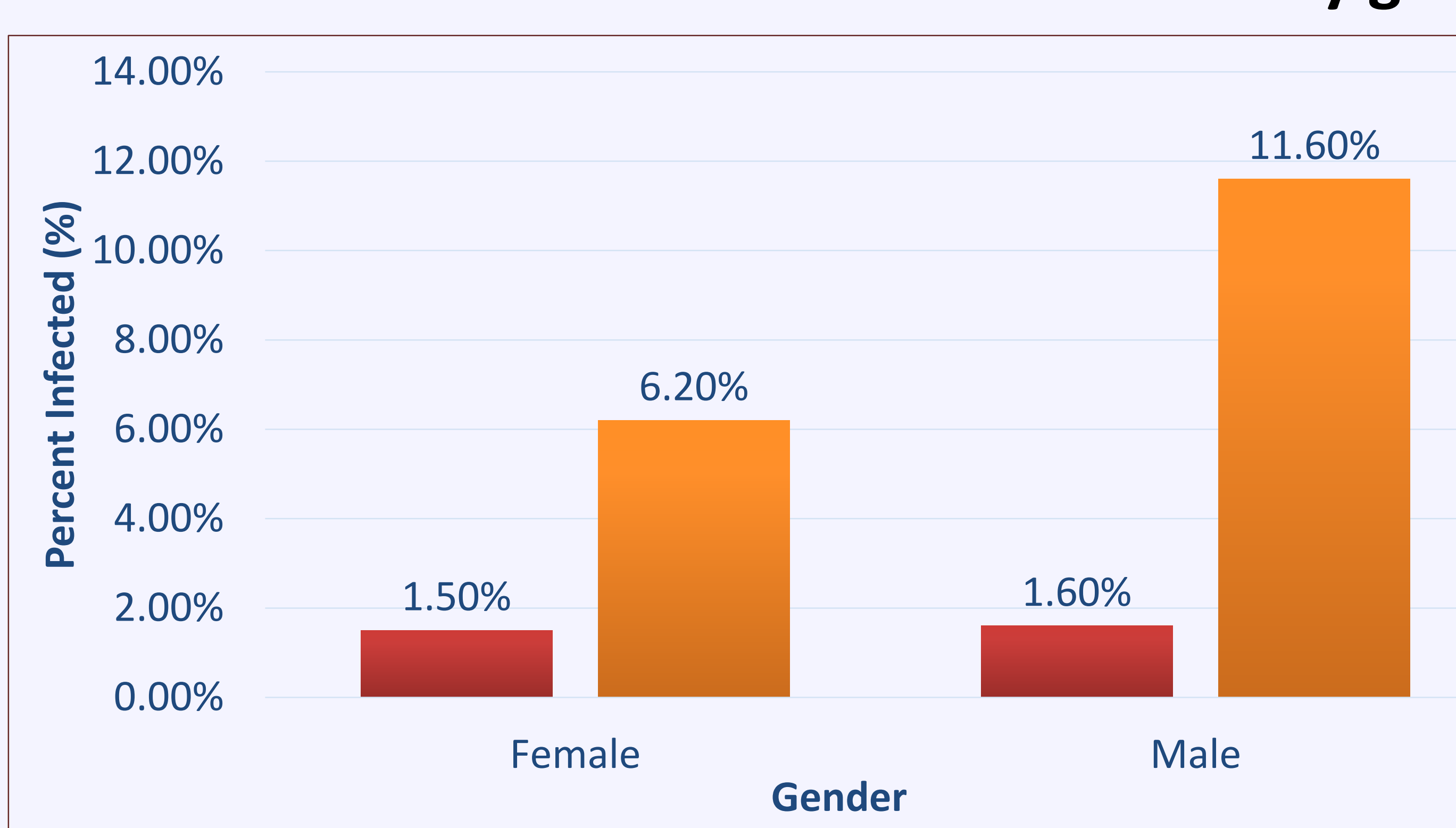

- Active COVID-19 Infection (RT-PCR) « Prevalence of COVID-19 Infection (Blood IgG)

Figure 2: this figure shows the percentage of COVID-19 prevalence and active infection by gender. Active cases were Almost similar across both genders (1.5\%-1.6\%), while IgG prevalence was higher in males (11.6 $\%)$ compared with females $(6.2 \%)$

\section{Prevalence and active COVID-19 infection by nationality.

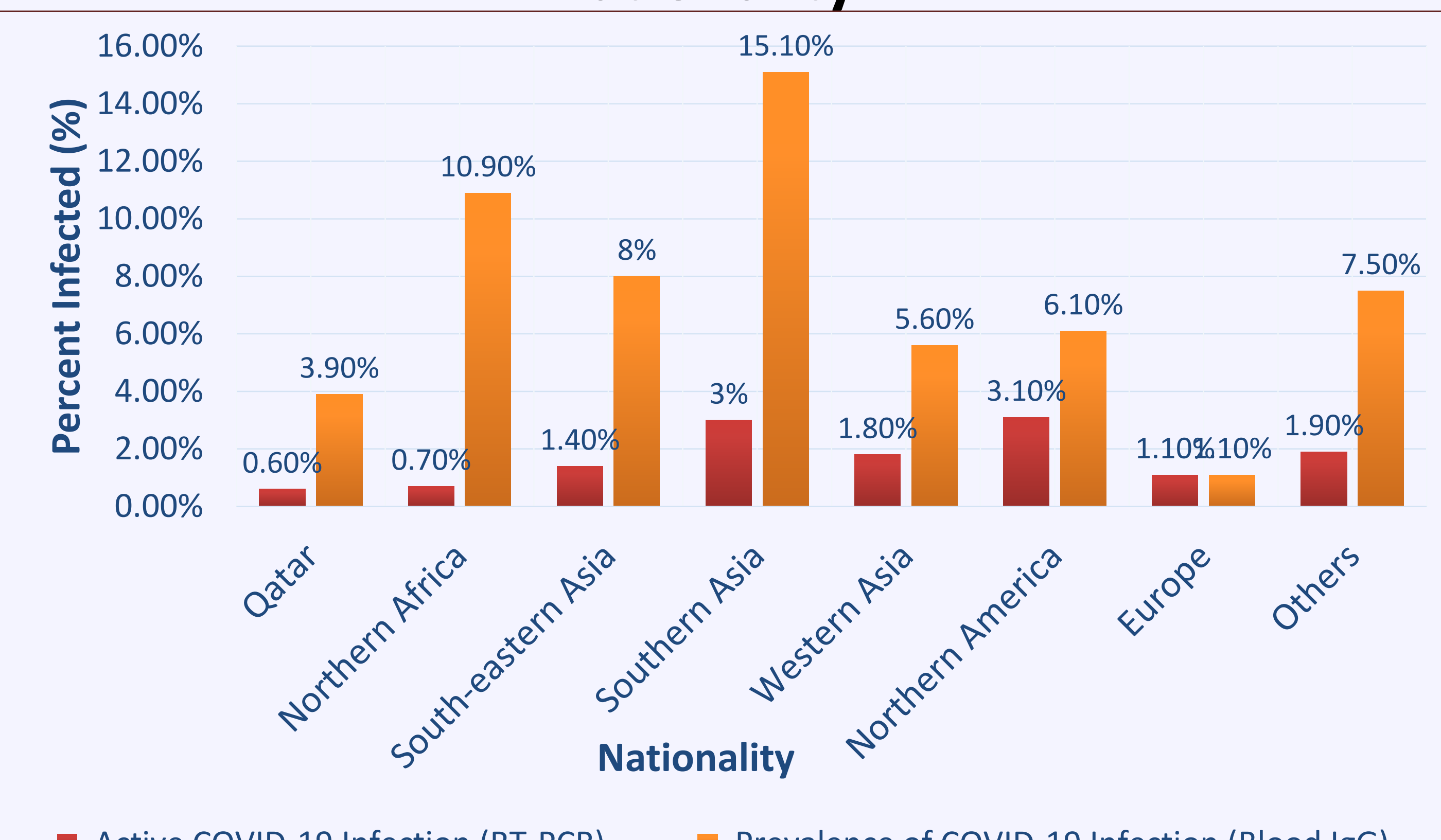

Figure 3: this figure shows the percentage of COVID-19 prevalence and active infection among nationalities in Qatar. Active Covid-19 infection was higher amongst North American (3.1\%) and Southern Asian (3\%) nationalities. While North African (10.9\%) and Southern Asian (15.1\%) nationalities had higher $\operatorname{IgG}$ prevalence

\section{Prevalence and active COVID-19 infection by} municipality

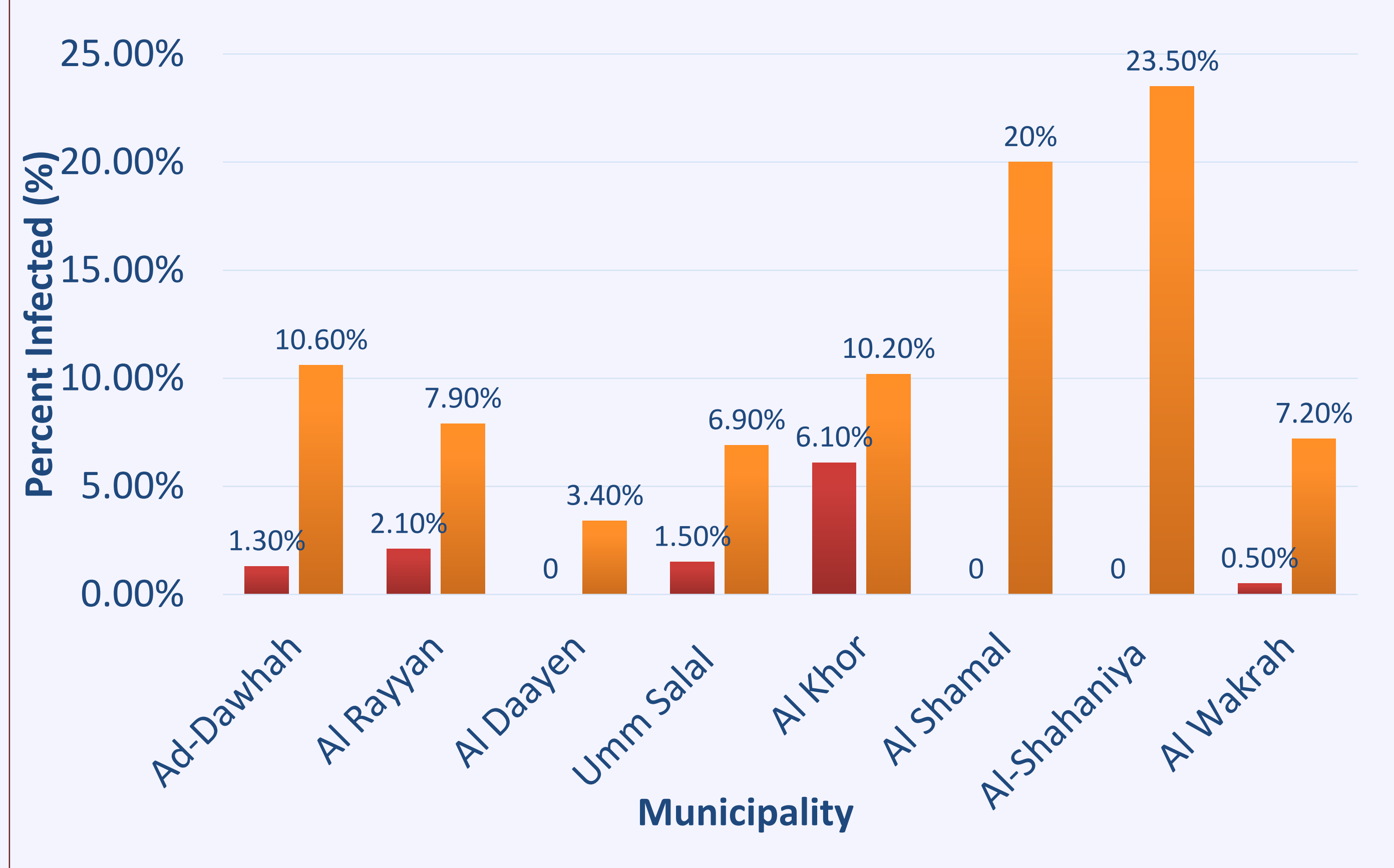

Figure 4: this figure shows the percentage of COVID-19 prevalence and active infection among municipalities. Highest active COVID-19 cases were in $\mathrm{Al} \mathrm{Khor} \mathrm{municipality} \mathrm{(6.1 \% ),} \mathrm{and} \mathrm{highest} \mathrm{prevalence} \mathrm{incidence}$ were Al Shahaniya (23.5 \%), Al Shamal (20\%), Ad Dawhah (10.6\%) and $\mathrm{Al} \mathrm{Khor} \mathrm{(10.2 \% )}$

Table 2: overall point and period prevalence of COVID-19 infection

\begin{tabular}{|c|c|c|c|c|c|c|c|c|c|}
\hline \multicolumn{2}{|c|}{ Previous RT-PCR } & \multicolumn{2}{|c|}{ Current RT-PCR } & \multicolumn{2}{|c|}{ Serology ${ }^{\ddagger}$} & \multicolumn{2}{|c|}{$\begin{array}{c}\text { Point } \\
\text { Prevalence }\end{array}$} & \multicolumn{2}{|c|}{$\begin{array}{c}\text { Period } \\
\text { Prevalence }\end{array}$} \\
\hline $\begin{array}{l}\text { Positive } \\
\text { Results }\end{array}$ & Total & $\begin{array}{l}\text { Positive } \\
\text { Results }\end{array}$ & Total & $\begin{array}{l}\text { Positiv } \\
\text { e } \\
\text { Result } \\
\text { s }\end{array}$ & Total & $\%$ & $95 \% \mathrm{Cl}$ & $\%$ & $95 \% \mathrm{Cl}$ \\
\hline$N(\%)$ & $\mathrm{N}$ & $N(\%)$ & $\mathrm{N}$ & $N(\%)$ & $\mathrm{N}$ & & & & \\
\hline $61(2.9 \%)$ & 2084 & 32 (1.6) & 2061 & $\begin{array}{l}187 \\
(9.1)\end{array}$ & 2052 & 1.6 & $1.1-2.2$ & 9.5 & $8.2-10.8$ \\
\hline
\end{tabular}

\section{Conclusion}

In conclusion, the overall point prevalence and period prevalence of SARS-CoV2 was 1.6 and $9.5 \%$ respectively. The findings suggest significant asymptomatic SARSCoV2 infection in the population. More importantly, despite the significant number of RT-PCR tests undertaken in the country, the study found a considerable difference in highlights the limitations of using RT-PCR as a screening tool unless undertaken using a robust sampling methodology. As the study was designed to represent Qatar's population, there was notable differences in period prevalence of SARS-CoV2 infection by age, gender, nationality and municipality.

\section{References}

